\title{
Silencing of miR-1-1 and miR-133a-2 cluster expression by DNA hypermethylation in colorectal cancer
}

\author{
WEI-SHONE CHEN ${ }^{1}$, CHUNG-MAN LEUNG ${ }^{2}$, HUNG-WEI PAN ${ }^{3}$, LING-YUEH HU ${ }^{4}$, \\ SUNG-CHOU LI ${ }^{5}$, MENG-RU HO ${ }^{5}$ and KUO-WANG TSAI ${ }^{3,6}$
${ }^{1}$ Department of Surgery, Veterans General Hospital, Taipei; Departments of ${ }^{2}$ Radiation Oncology and ${ }^{3}$ Medical Education and Research, Kaohsiung Veterans General Hospital, Kaohsiung; ${ }^{4}$ Institute of Biomedical Sciences and ${ }^{5}$ Genomics Research Center, Academia Sinica, Taipei; ${ }^{6}$ Department of Biotechnology,
Tajen University, Pingtung, Taiwan, R.O.C.

Received April 10, 2012; Accepted June 4, 2012

DOI: 10.3892/or.2012.1899

\begin{abstract}
MicroRNAs are small non-coding RNA molecules that play important roles in the multistep process of colorectal carcinoma (CRC) development. The present study evaluated the relationship between miR-1-1 and miR-133a-2 expression and DNA methylation, and its putative biological role in CRC. The results indicated that DNA methylation regulated the expression of the miR-1-1 and miR-133a-2 cluster in CRC cell lines. Expression of miR-1 and miR-133a was further evaluated in 64 paired tissue samples (CRC tumor and adjacent normal mucosa) using the stem-loop real-time polymerase chain reaction. The miR-1-133a cluster displayed significantly lower expression in CRC tissue compared to adjacent normal mucosa $(\mathrm{P}<0.001)$. The results also indicated frequent hypermethylation of the $\mathrm{CpG}$ islands upstream of miR-1-133a (54.6\%). Liver metastatic tissues exhibited significantly lower miR-1 $(\mathrm{P}<0.001)$ and miR-133a $(\mathrm{P}<0.001)$ expression compared to adjacent normal mucosa. Expression of the miR-1-133a cluster inversely correlated with TAGLN2 in the tumor specimens. In conclusion, epigenetic repression of the miR-1-133a cluster may play a critical role in colorectal cancer metastasis by silencing TAGLN2.
\end{abstract}

\section{Introduction}

Colorectal cancer (CRC) is one of the major causes of cancerrelated death worldwide (1). Metastasis to regional lymph nodes represents a major problem, usually leading to poor survival rate after radical operation (2). The investigation of

Correspondence to: Dr Kuo-Wang Tsai, Department of Medical Education and Research, Kaohsiung Veterans General Hospital, Kaohsiung 813, Taiwan, R.O.C.

E-mail: kwtsai@vghks.gov.tw

Key words: microRNA, colon cancer, DNA methylation, miR-1, miR-133a metastasis-associated genes for the early detection or therapeutic targeting of CRC could, therefore, potentially improve the survival rate of future $\mathrm{CRC}$ patients.

MicroRNAs are non-coding small RNAs (18-22 nucleotides in size) and negatively regulate gene expression by binding to the 3'UTR of target genes (3). They are involved in a variety of biological processes including differentiation, morphogenesis, proliferation, and apoptosis (3). Studies have reported dysregulation of miRNAs in a number of human diseases including colon cancer $(4,5)$. Several miRNAs display tumor suppressive functions while others have an oncogenic role during carcinogenesis (5-7). Investigators have linked the levels of expression of these miRNAs to clinicopathological features of disease. Analysis of their expression might, therefore, facilitate the detection and diagnosis of cancer (8-10). The DNA methylation of gene promoters is critical in the repression of gene expression in various cell types or development stages. Abnormal methylation of tumor suppressor genes plays a significant role in tumor development (11-15). Accumulating evidence has shown that several tumor-associated miRNAs are overexpressed or downregulated during CRC progression. Among these dysregulated miRNAs, tumor suppressive miRNAs, including miR-9, miR-129, miR-134, miR-342 and miR-34b/c, are frequently silenced by aberrant DNA hypermethylation in CRC genomic DNA (16-19).

The miR-1 and miR-133a family of miRNAs is encoded at two paralogous loci and reportedly downregulates and displays tumor suppressive function in various human cancers, including bladder cancer, prostate cancer, lung cancer, esophageal squamous carcinoma and colon cancer (20-24). Datta et al (25) reported that DNA methylation can mediate the miR-1 gene and suppress tumor cell growth by repressing its oncogenic targets MET, FoxP1 and HDAC4 in hepatocellular carcinoma. However, the relationships between the DNA methylation status of the miR-1-133a cluster, levels of expression and biological function in CRC have yet to be fully elucidated. The present study performed a series of sequential analyses to evaluate the methylation status and expression of the miR-1-133a cluster in 64 paired samples of CRC tissue and 14 liver metastatic tissue samples. Results indicated that 
high frequency DNA hypermethylation leads to silencing of the miR-1-133a cluster expression, and that this silencing is associated with tumor metastasis.

\section{Materials and methods}

Clinical samples. Sixty-four paired tumor and adjacent normal mucosa samples and 14 metastatic liver tumor, primary tumor and adjacent mucosa samples were obtained from CRC patients who underwent surgical operation at the Department of Surgery, Veterans General Hospital, Taipei, Taiwan. Informed consent was obtained from all patients.

Extraction of RNA. The total RNA of the tissue was extracted using TRIzol reagent (Invitrogen, Carlsbad, CA, USA) according to the instruction manual. Briefly, tissue samples were homogenized in $1 \mathrm{ml}$ TRIzol reagent and mixed with $0.2 \mathrm{ml}$ chloroform to extract protein, before RNA was precipitated using $0.5 \mathrm{ml}$ isopropanol. The concentration, purity and amount of total RNA were determined using a Nanodrop 1000 spectrophotometer (Nanodrop Technologues, Inc., USA).

Cell lines and 5-Aza-dC treatment. Human colorectal cancer cell lines, HT29 and B77, were obtained from the American Type Culture Collection and maintained in Dulbecco's modified Eagle's medium supplemented with 10\% inactivated FBS (Invitrogen). Colorectal cancer cells were cultured in the presence or absence of $2.5 \mu \mathrm{M} 5$-Aza-dC for 4 days. The total RNA was then prepared using TRIzol (Invitrogen), according to the previously described protocol.

Real-time polymerase chain reaction and statistical analysis. The primers were designed to the mature miRNAs for miR-1 and miR-133a for reverse transcription according to the methods described by Chen et al (26). One microgram total RNA was reverse-transcribed using a stem-loop RT reaction with RT primers and SuperScript III Reverse Transcriptase according to the user's manual (Invitrogen). The reaction was performed with the following incubation conditions: $30 \mathrm{~min}$ at $16^{\circ} \mathrm{C}$, followed by 50 cycles of $20^{\circ} \mathrm{C}$ for $30 \mathrm{sec}, 42^{\circ} \mathrm{C}$ for $30 \mathrm{sec}$ and $50^{\circ} \mathrm{C}$ for $1 \mathrm{sec}$. The enzyme was subsequently inactivated by incubation at $85^{\circ} \mathrm{C}$ for $5 \mathrm{~min}$. Real-time PCR reactions were performed using a microRNA-specific forward primer and a universal reverse primer, and were conducted at $94^{\circ} \mathrm{C}$ for $10 \mathrm{~min}$, followed by 40 cycles of $94^{\circ} \mathrm{C}$ for $15 \mathrm{sec}$ and $60^{\circ} \mathrm{C}$ for $32 \mathrm{sec}$. Gene expression was detected using a SYBR-Green I assay (Applied Biosystems, Foster City, CA, USA) and the expression levels of miRNAs were normalized to that of U6. Expression of TAGLN1 and LASP1 was examined using real-time polymerase chain reaction (PCR) with a gene-specific primer and normalized to GAPDH. All reactions were performed in triplicate and the differences between tissue types were analyzed using Student's t-test. The difference was considered to be significant at P-value $<0.05$.

The individual primers used were as follows: miR-1-RT, 5'-CTCAACTGGTGTCGTGGAGTCGGCAATTCAGTTGA GATACATAC-3'; miR-133a-RT, 5'-CTCAACTGGTGTCG TGGAGTCGGCAATTCAGTTGAGCAGCTGGT-3'; miR1-GSF, 5'-CGGCGGTGGA ATGTAAAGA AGT-3'; miR-133a-GSF, 5'-CGGCGGTTTGGTCCCCTTCAAC-3';
Universal-R, 5'-CTGGTGTCGTGGAGTCGGCAATTC-3'; U6-F, 5'-CTCGCTTCGGCAGCACA-3'; U6-R, 5'-AACG CTTCACGAATTTGCGT-3'; TAGLN1-F, 5'-CGAGGG GCAGGCCCCAGTAA-3'; TAGLN1-R, 5'-GTCCGCTG CACACAGGCCAT-3'; LASP1-F, 5'-GCAACAGAGTGAG CTCCAGAG-3'; LASP1-R, 5'-TGAAACCTTTGCCCTTG TTC-3'; GAPDH-F, 5'-TGCACCACCAACTGCTTAGC-3'; GAPDH-R, 5'-GGCATGGACTGTGGTCATGAG-3'.

Conversion of DNA bisulfite and COBRA analysis. Genomic DNA was extracted from cultured cells or CRC tissues using TRIzol reagent (Invitrogen) and then $2 \mu \mathrm{g}$ genomic DNA was subjected to bisulfite conversion using the EZ DNA Methylation-Gold kit (Zymo Research Corp., Orange, CA, USA). The conditions for the bisulfite conversion reaction were $98^{\circ} \mathrm{C}$ for $10 \mathrm{~min}$ followed by $64^{\circ} \mathrm{C}$ for $2.5 \mathrm{~h}$, with a final incubation at $4^{\circ} \mathrm{C}$ for up to $20 \mathrm{~h}$ in a PCR thermocycler. The modified genomic DNA was used for the methylation analysis of $\mathrm{CpG} 25$, CpG81 and $\mathrm{CpG} 26$ using the specific methylation primers. The PCR conditions were as follows: $94^{\circ} \mathrm{C}$ for $10 \mathrm{~min}$, followed by 35 cycles of $94^{\circ} \mathrm{C}$ for $1 \mathrm{~min}, 60^{\circ} \mathrm{C}$ for $1 \mathrm{~min}$ and $72^{\circ} \mathrm{C}$ for $30 \mathrm{sec}$, with a final extension at $72^{\circ} \mathrm{C}$ for $10 \mathrm{~min}$ using a PCR thermocycler and HotStart Taq DNA polymerase (Qiagen, Hilden, Germany). The methylation status of the genomic DNA of individual samples was also examined using BstUI digestion (New England Biolabs, MA, USA). The digested PCR fragments were then separated on $2 \%$ agarose gel. The individual primers used were as follows: CpG25-F, 5'-GGA GGGGTAGGATAGTAGTTTGAGT-3'; CpG25-R, 5'-AAAA AAACCTAACCTAAAAAACCAAAA-3'; CpG81-F, 5'-GGT GAGTTTTGTTTAGTTTATTATTAT-3'; CpG81-R, 5'-ATC AAAATTCCTACCTCCCAACTA-3'; CpG26-F, 5'-TGTTTG TGTTAGTAGGTGGAAGTGT-3'; CpG26-R, 5'-CCTCTA AACAATTTCTACCCTAACC-3'.

\section{Results}

Transcriptional activity of the miR-1-133a cluster is controlled by DNA methylation. Expression of miR-1 and miR-133a originated from two genomic loci in chromosome 18 and chromosome 20. This study investigated the DNA methylation alterations to regions upstream of miR-1 and miR-133a. Searches of the UCSC database identified $4 \mathrm{CpG}$ islands located at the putative transcription start sites of the miR-1-1 and miR-133a-2 loci, which suggested that DNA methylation might control their transcriptional activities (Fig. 1A). The methylation status of $3 \mathrm{CpG}$-rich regions in 2 human CRC cell lines was analyzed using a COBRA approach. Complete hypermethylation of $\mathrm{CpG}$ of the $\mathrm{CpG} 81$ occurred in 2 human CRC cell lines (Fig. 1C). After 4 days of treatment with 5-Aza$\mathrm{dC}$, the transcriptional activities of miR-1 and miR-133a had reactivated in examined cells (Fig. 1B). These results indicated that the human miR-1-133a cluster can be regulated epigenetically by DNA methylation in CRC cells.

Concurrent silencing of miR-1 and miR-133a by DNA hypermethylation in colon cancer. Further analysis of miR-1 and miR-133a expression in primary CRC tissues of 64 patients revealed the significant downregulation of mature miR-1 and miR-133a expression compared to expression in normal 
A

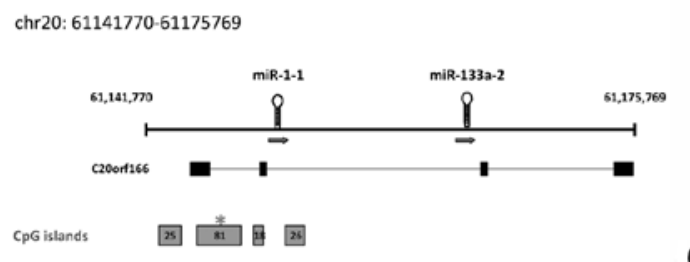

CpG25

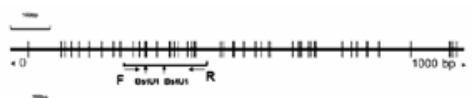

CpG81:

CpG26:
B

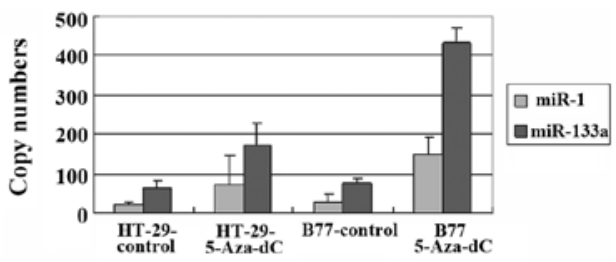

C

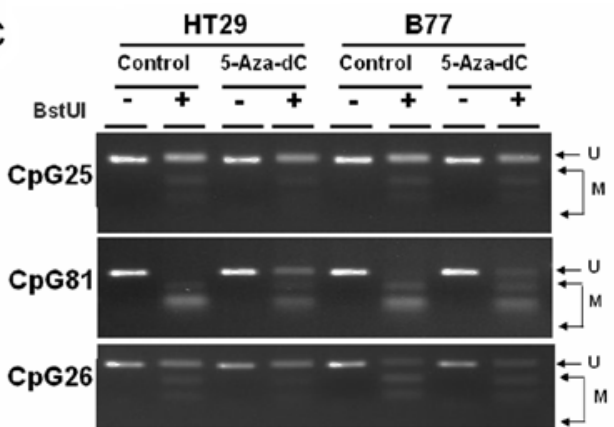

Figure 1. Silencing of miR-1-1 and miR-133a-2 cluster expression by DNA methylation in colon cancer cells (A) Schematic representation of the locations of the miR-1 and miR-133a genes. Expressed sequence tag (EST) transcripts were identified from the UCSC website (http://genome.ucsc.edu/). Stem-loops indicate the positions of miR-1 and miR-133a; blocks indicate their upstream CpG islands. Asterisks indicate the analyzed CpG islands. (B) Expression of miR-1-1 and miR-133a was analyzed using real-time PCR in human colon cancer cells. The U6 snRNA was used as the internal control with gene expression calculated relative to the internal control $(\Delta \mathrm{Ct})$. The number of copies of each miRNA was estimated using the standard equation $10000 \times\left(2^{-\Delta C}\right)$. (C) The methylation status of each CpG island upstream of the miR-1-1 and miR-133a-2 cluster was analyzed using the COBRA approach in HT-29 and B77 cells. Arrows indicate the unmethylated (u)/methylated $(\mathrm{m})$ alleles.
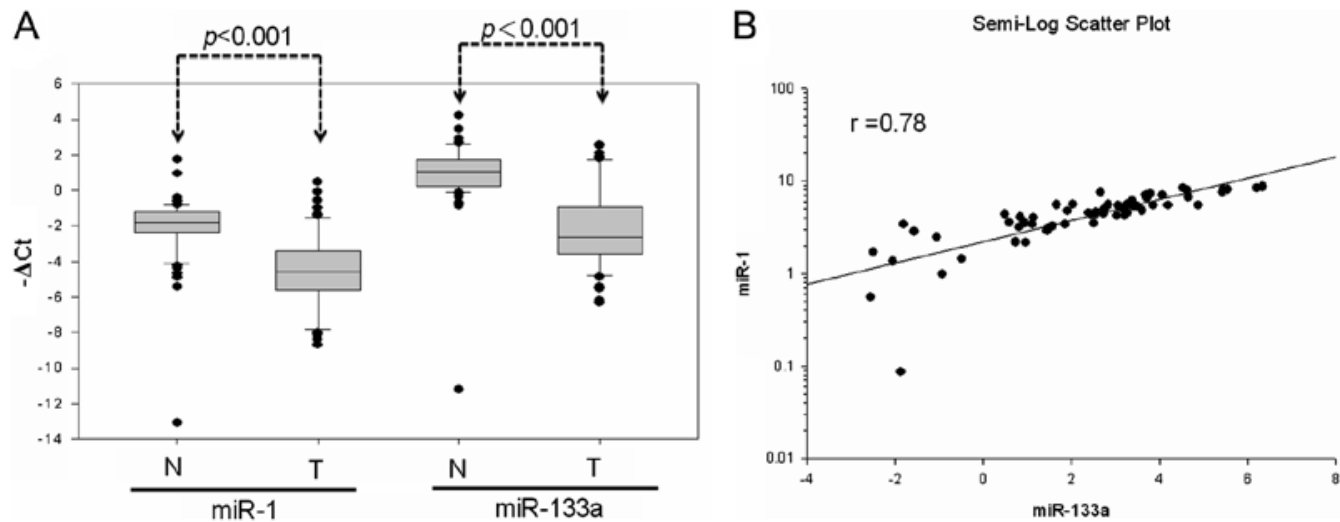

Figure 2. Concurrent downregulation of miR-1 and miR-133a expression in 64 CRC patients (A) Expression of miR-1 and miR-133a in the CRC tissues from 64 patients was analyzed using quantitative stem-loop PCR with U6 as an internal control. All samples were assessed in triplicate and analyzed using Student's $\mathrm{t}$-test $(\mathrm{P}<0.05$ was considered significant). (B) Correlation between miR-1 and miR-133a expression in 64 clinical samples. The correlation coefficient of 0.78 with $\mathrm{P}<0.001$ indicated that miR-1 expression was highly correlated with that of miR-133a.

mucosa (miR-1, 55 out of 64; miR-133a, 56 out of 64) (Fig. 2A). Simultaneous miR-1 and miR-133a downregulation occurred at a high frequency in the examined samples $(84.3 \% ; 54$ out of 64). This showed that miR-1 expression well-correlated with that of miR-133a in the 64 CRC patients ( $r=0.78$ ) (Fig. 2B). These results suggested that DNA hypermethylation led to low miR-1-133a cluster expression in colon cancer cells. Further analyses evaluated whether downregulation of the miR-1-133a cluster resulted from DNA hypermethylation of CpG-rich regions upstream of miR-1-1 and miR-133a-2. Analysis of the methylation status of CpG81 using COBRA analysis revealed that tumor-specific DNA methylation of $\mathrm{CpG} 81$ occurred in 35 out of $64(54.6 \%)$ of the primary CRC samples (Fig. 3A).
Expression of miR-1 $(\mathrm{P}<0.01)$ and miR-133a $(\mathrm{P}<0.001)$ was significantly reduced in DNA hypermethylation compared with DNA hypomethylation cases of primary colon cancer tissues (Fig. 3B). These results indicated that abnormal DNA hypermethylation concurrently silenced miR-1 and miR-133a expression in CRC.

Expression of the miR-1-133a cluster is associated with colon cancer metastasis. Expression of the miR-1-133a cluster in primary tumor, metastatic liver tumor and the corresponding normal mucosa of 14 CRC patients was evaluated using real-time PCR analysis. Relatively lower miR-1 expression occurred in 10 of 14 (71.4\%) primary tumors and in 12 of 14 


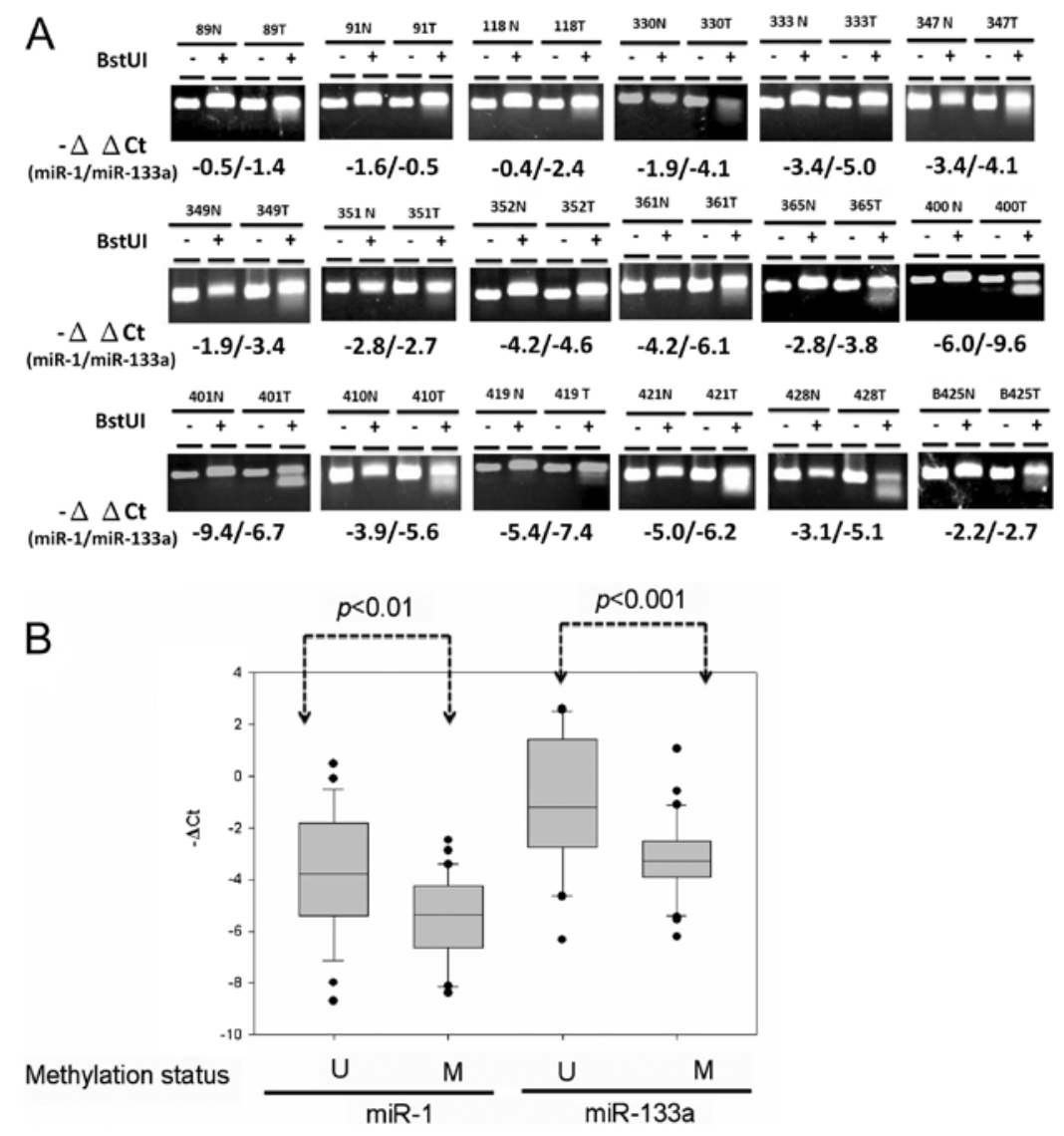

Figure 3. Silencing of miR-1-133a cluster expression by DNA hypermethylation in 64 CRC patients. (A) The methylation status of CpG81, upstream of miR-1-1 and miR-133a-2 in the colorectal carcinoma genome, was analyzed using COBRA. Fold changes in miR-1 and miR-133a expression in CRC tissues are indicated by the $\Delta \Delta \mathrm{Ct}$ compared to adjacent normal tissue $(\Delta \Delta \mathrm{Ct}=\Delta \mathrm{Ct}$, tumor $-\Delta \mathrm{Ct}$, adjacent normal). Asterisks indicate higher methylation status in the genomic DNA of the colorectal tumor in comparison with that of adjacent normal mucosa. (B) Expression of miR-1 and miR-133a in 64 patients (duplicated from Fig. 2). Comparison between their expression in DNA hypermethylation and hypomethylation states in primary colon cancer tissues.

$(85.7 \%)$ metastatic liver tumors in comparison to the corresponding normal mucosa from the same patients. Similarly, significantly lower miR-133a expression occurred in 12 of 14 $(85.7 \%)$ primary tumors and in 14 of $14(100 \%)$ metastatic liver tumors in comparison to the corresponding normal mucosa. Expression of miR-1 was significantly lower in the primary tumor $(\mathrm{P}<0.05)$ and metastatic liver tumor $(\mathrm{P}<0.001)$ than in adjacent normal mucosa; miR-133a expression was also significantly lower in the primary tumor $(\mathrm{P}<0.001)$ and metastatic liver tumor $(\mathrm{P}<0.001)$ than in adjacent normal mucosa (Fig. 4). These data suggested the involvement of miR-1-133a cluster expression in CRC metastasis.

Reduced repression of TAGLN2 resulting from DNA hypermethylation of miR-1 and miR-133a. Identification of the target genes of miR-1 and miR-133a is important for the investigation of their biological functions. The previously described data indicated that DNA hypermethylation concurrently silenced miR-1 and miR-133a expression in the CRC genome, and that low expression of miR-1 and miR-133a might contribute to CRC invasion and metastasis. This suggests that miR-1 and miR-133a might share similar biological functions in the suppression of cancer cell migration during CRC progression. The putative targets of miR-1 and miR-133a were identified using the target prediction program (http://www.ebi.

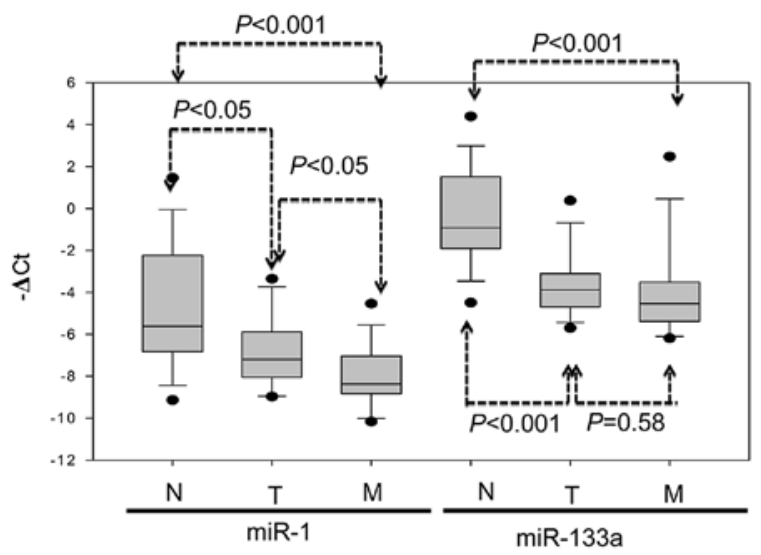

Figure 4. Expression of miR-1 and miR-133a in liver metastasis patients Levels of miR-1 and miR-133a expression in primary tumor, metastatic liver tumor and the corresponding normal mucosa of 14 CRC patients were examined using quantitative stem-loop PCR with U6 as an internal control. All samples were assessed in triplicate and analyzed using Student's t-test $(\mathrm{P}<0.05$ was considered significant).

ac.uk/enright-srv/microcosm/htdocs/targets/v5/). As shown in Table I, this provided 49 putative targets for simultaneous silencing by miR-1 and miR-133a. The targets TAGLN2 and LASP1, which may be involved in promoting cancer migration, 
Table I. Identify putative targets of miR-1-133a through target prediction program (MicroCosm Targets Version 5).

\begin{tabular}{|c|c|}
\hline ABL2 & v-abl Abelson murine leukemia viral oncogene homolog 2 (arg, Abelson-related gene) \\
\hline ASH1L & ash1 (absent, small, or homeotic)-like (Drosophila) \\
\hline BCL11A & B-cell CLL/lymphoma 11A (zinc finger protein) \\
\hline CALM1 & Calmodulin 1 (phosphorylase kinase, $\delta$ ) \\
\hline CAP1 & CAP, adenylate cyclase-associated protein 1 (yeast) \\
\hline CORO1C & Coronin, actin binding protein, $1 \mathrm{C}$ \\
\hline CREB5 & cAMP responsive element binding protein 5 \\
\hline CTBP2 & C-terminal binding protein 2 \\
\hline DDX3X & DEAD (Asp-Glu-Ala-Asp) box polypeptide 3, X-linked \\
\hline EIF2C1 & Eukaryotic translation initiation factor $2 \mathrm{C}, 1$ \\
\hline EVI1 & Ecotropic viral integration site 1 \\
\hline EYA4 & Eyes absent homolog 4 (Drosophila) \\
\hline FOXP1 & Forkhead box P1 \\
\hline GABBR2 & $\gamma$-aminobutyric acid (GABA) B receptor, 2 \\
\hline GCLC & Glutamate-cysteine ligase, catalytic subunit \\
\hline $\mathrm{HIC} 2$ & Hypermethylated in cancer 2 \\
\hline HIVEP2 & Human immunodeficiency virus type I enhancer binding protein 2 \\
\hline LASP1 & LIM and SH3 protein 1 \\
\hline LASS2 & Ceramide synthase 2 \\
\hline LETM1 & Leucine zipper-EF-hand containing transmembrane protein 1 \\
\hline LIN7C & Lin-7 homolog $\mathrm{C}$ \\
\hline MAP3K3 & Mitogen-activated protein kinase kinase kinase 3 \\
\hline MEIS1 & Meis homeobox 1 \\
\hline MKL2 & MKL/myocardin-like 2 \\
\hline NFAT5 & Nuclear factor of activated T-cells 5 \\
\hline PDIK1L & PDLIM1 interacting kinase 1 like \\
\hline PFN2 & Profilin 2 \\
\hline PIK3C2A & Phosphoinositide-3-kinase, class $2, \alpha$ polypeptide \\
\hline PREX1 & Phosphatidylinositol-3,4,5-trisphosphate-dependent Rac exchange factor 1 \\
\hline PTBP1 & Polypyrimidine tract binding protein 1 \\
\hline QKI & QKI, KH domain containing, RNA binding \\
\hline RARB & Retinoic acid receptor, $\beta$ \\
\hline RICTOR & RPTOR independent companion of MTOR, complex 2 \\
\hline SGK269 & NKF3 kinase family member \\
\hline SH3PXD2B & SH3 and PX domains 2B \\
\hline SLC25A36 & Solute carrier family 25 , member 36 \\
\hline SP1 & Sp1 transcription factor \\
\hline SYT1 & Synaptotagmin I \\
\hline TAGLN2 & Transgelin 2 \\
\hline TFE3 & Transcription factor binding to IGHM enhancer 3 \\
\hline TTPAL & Tocopherol $(\alpha)$ transfer protein-like \\
\hline UBXD8 & Fas associated factor family member 2 \\
\hline VAMP2 & Vesicle-associated membrane protein 2 \\
\hline VAT1 & Vesicle amine transport protein 1 homolog \\
\hline WIPF2 & WAS/WASL interacting protein family, member 2 \\
\hline YES1 & v-yes-1 Yamaguchi sarcoma viral oncogene homolog 1 \\
\hline YPEL2 & Yippee-like 2 \\
\hline ZBTB4 & Zinc finger and BTB domain containing 4 \\
\hline ZNF217 & Zinc finger protein 217 \\
\hline
\end{tabular}

were selected for further analysis (27,28). Previous studies have shown that miR-1 and miR-133a can repress the expression of TAGLN2 and LASP1 by targeting the 3'UTR region $(20,24)$. Expression of TAGLN2 and LASP1 in paired adjacent normal and gastric tumor tissues from 64 patients was, thus, further examined using real-time quantitative PCR (Fig. 5). Results indicated an inverse correlation between TAGLN2 mRNA and miR-1-133a cluster expression in the tumor specimens. The 


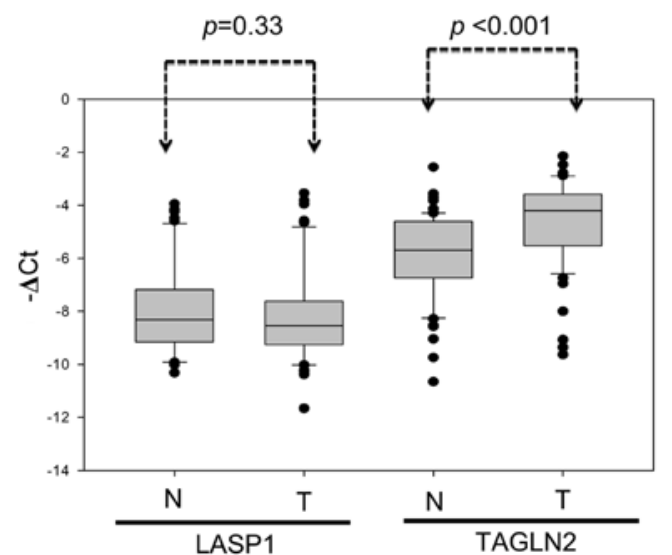

Figure 5. Expression of TAGLN2 and LASP1 in 64 CRC patients. Expression of TAGLN2 and LASP1 in the CRC tissues from 64 patients was analyzed using real-time PCR with GAPDH as an internal control. All samples were assessed in triplicate and analyzed using Student's t-test $(\mathrm{P}<0.05$ was considered significant).

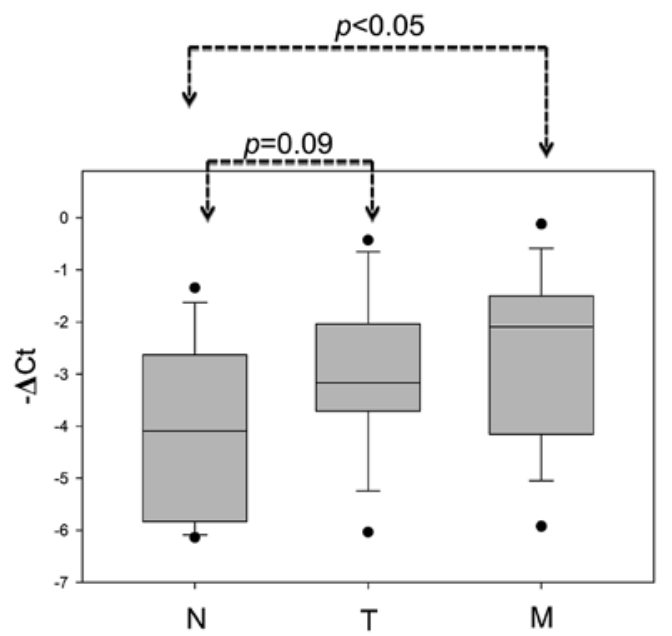

Figure 6. Expression of TAGLN2 in liver metastasis patients. Expression of TAGLN2 was examined in primary tumor, metastatic liver tumor and the corresponding normal mucosa of 14 CRC patients using real-time RT-PCR with GAPDH as an internal control. All samples were assessed in triplicate and analyzed using Student's t-test ( $\mathrm{P}<0.05$ was considered significant).

TAGLN2 mRNA levels were significantly higher in the CRC tissues than in the adjacent normal mucosa (Fig. 5, $\mathrm{P}<0.001$ ). In contrast, miR-1-133a cluster expression was significantly lower in the tumor tissues than in normal adjacent mucosa (Fig. 2A; $\mathrm{P}<0.001)$. The metastatic liver tumors also displayed significantly increased TAGLN2 expression in comparison to the corresponding normal mucosa from the same patient (Fig. 6; $\mathrm{P}<0.05)$. In summary, DNA hypermethylation in miR-1-1 and miR-133a-2 loci resulted in their low expression and TAGLN2 overexpression. This process might have a significant role in colorectal cancer metastasis.

\section{Discussion}

Colorectal carcinogenesis is a multistep process involving the genetic dysregulation of oncogenes and tumor suppressor genes. Recent studies have shown that microRNA-associated transcriptionally regulated gene expression plays a critical role in the control of various cellular functions, such as cell proliferation, cell cycle, apoptosis and cell motility (5-7). Investigators have described miR-1 and miR-133a as regulators of muscle cell growth and differentiation, and identified that abnormal miR-1-133a cluster expression contributes to cardiovascular disease (29-31). Recent studies have also described downregulation of miR-1 expression and its tumor suppressor activity in human cancers, including prostate cancer, hepatocellular carcinoma, bladder urothelial carcinoma, esophageal squamous cell carcinoma, lung cancer, rhabdomyosarcoma and colorectal carcinoma. In addition, miR-1 acts as a tumor suppressor by suppressing oncotargets in human cancer, including Met, Foxp1, HDAC4, F-actin, TAGLN2, SRSF9, fibronectin and LASP1 (25,32-37). Several studies have identified the downregulation of miR-133a expression in cancers and suggested that miR-133a has a tumor suppressive function in esophageal squamous cell carcinoma and bladder, head, breast and lung cancers by repressing FSCN1, GSTP1, MSN, ARPC5 and CAV1 (38-42). Some studies have also reported the concurrent silencing of miR-1 and miR-133a in prostate cancer, squamous cell carcinoma and bladder cancer $(20-22,24)$. The restoration of miR-1 and miR-133a in the bladder cells led to significant inhibition of cell proliferation and migration by way of repression of TAGLN2 and LASP1 expression $(20,24)$. Nohata et al observed similar trends in squamous cell carcinoma; identifying that miR-1 and miR-133a overexpression inhibited cell proliferation and induced apoptosis by regulating TAGLN2 and PNP (22). In the present study, miR-1 ( $<<0.01)$ and miR-133a $(\mathrm{P}<0.001)$ expression was significantly lower in liver metastatic tissue than in adjacent normal mucosa, suggesting that these miRNAs are crucially involved in colorectal cancer metastasis. Results indicated downregulation of the miR-1-133a cluster expression in $84.3 \%$ of the CRC samples (54 out of 64) and TAGLN2 overexpression in $83.3 \%$ of the samples (45 out of 54). The TAGLN2 protein is a member of the calponin family of actin-binding proteins. Several cancer types, including HCC, lung cancer and head and neck squamous cell carcinoma, exhibit TAGLN2 overexpression and promoted cancer cell migration and invasion $(22,27,43,44)$. Zhang et al $(27)$ further reported the correlation between TAGLN2 expression and lymph node metastasis in colorectal cancer. Overall, these data indicate that TAGLN2 might have an oncogenic function and promote cancer migration, which is regulated by the miR-1-133a cluster in CRC.

Aberrant DNA methylation is a common feature of colorectal carcinoma, leading to aberrant expression of tumor suppressive miRNAs through the hypermethylation of their promoters. Datta et al first observed DNA hypermethylation in miR-1-1 in human HCC cells and primary HCC, identifying that ectopic expression of DNA-hypermethylated miR-1-1 suppressed tumor cell growth by targeting MET, FoxP1 and HDAC4 (25). Hudson et al (32) also demonstrated the epigenetic silencing of miR-1 in human prostate cancer following 5-Aza-dC treatment. In the present study, results indicated the concurrent downregulation of miR-1 and miR-133a expression in colon cancer tissue compared to adjacent normal mucosa. Tumor genomic DNA displayed a high frequency of DNA hypermethylation compared with that of the adjacent normal 
mucosa (53.2\%; Fig. 3). During the preparation of this manuscript, Suzuki et al (45) and Migliore et al (33) both reported the frequent methylation of miR-1-1 in colorectal cancer, in which it might function as a tumor suppressor.

In conclusion, detailed analyses of DNA methylation status in the miR-1-1 and miR-133a-2 upstream regions identified that DNA hypermethylation concurrently represses miR-1 and miR-133a expression. This reduces the microRNA-associated repression of TAGLN2 in colorectal carcinoma. In the future, DNA hypermethylation of the miR-1-133a cluster, along with identification of their target genes, could provide a promising strategy for the treatment of patients with CRC.

\section{Acknowledgements}

This study was supported in part by research funding from National Sciences Council (NSC 99-2320-B-010-021) and Kaohsiung Veterans General Hospital (VGHKS 101-010 and VGHKS101-118).

\section{References}

1. Shike M, Winawer SJ, Greenwald PH, Bloch A, Hill MJ and Swaroop SV: Primary prevention of colorectal cancer. The WHO Collaborating Centre for the Prevention of Colorectal Cancer. Bull World Health Organ 68: 377-385, 1990.

2. Sasaki H, Miura K, Horii A, et al: Orthotopic implantation mouse model and cDNA microarray analysis indicates several genes potentially involved in lymph node metastasis of colorectal cancer. Cancer Sci 99: 711-719, 2008.

3. Bartel DP: MicroRNAs: genomics, biogenesis, mechanism, and function. Cell 116: 281-297, 2004.

4. Wu WK, Law PT, Lee CW, et al: MicroRNA in colorectal cancer: from benchtop to bedside. Carcinogenesis 32: 247-253, 2011.

5. Zhang B, Pan X, Cobb GP and Anderson TA: microRNAs as oncogenes and tumor suppressors. Dev Biol 302: 1-12, 2007.

6. Babashah S and Soleimani M: The oncogenic and tumour suppressive roles of microRNAs in cancer and apoptosis. Eur J Cancer 47: 1127-1137, 2011.

7. Lima RT, Busacca S, Almeida GM, Gaudino G, Fennell DA and Vasconcelos MH: MicroRNA regulation of core apoptosis pathways in cancer. Eur J Cancer 47: 163-174, 2011.

8. Wilmott JS, Zhang XD, Hersey P and Scolyer RA: The emerging important role of microRNAs in the pathogenesis, diagnosis and treatment of human cancers. Pathology 43: 657-671, 2011

9. Luo X, Burwinkel B, Tao S and Brenner H: MicroRNA signatures: novel biomarker for colorectal cancer? Cancer Epidemiol Biomarkers Prev 20: 1272-1286, 2011.

10. Dhayat S, Mardin WA, Mees ST and Haier J: Epigenetic markers for chemosensitivity and chemoresistance in pancreatic cancer a review. Int J Cancer 129: 1031-1041, 2011.

11. Kim TY, Jong HS, Jung Y, Kim TY, Kang GH and Bang YJ: DNA hypermethylation in gastric cancer. Aliment Pharmacol Ther 20 (Suppl 1): S131-S142, 2004.

12. Oue N, Kuraoka K, Kuniyasu H, et al: DNA methylation status of hMLH1, p16(INK4a), and CDH1 is not associated with mRNA expression levels of DNA methyltransferase and DNA demethylase in gastric carcinomas. Oncol Rep 8: 1085-1089, 2001.

13. Momparler RL: Epigenetic therapy of cancer with 5-aza-2'-deoxycytidine (decitabine). Semin Oncol 32: 443-451, 2005.

14. Kozaki K, Imoto I, Mogi S, Omura K and Inazawa J: Exploration of tumor-suppressive microRNAs silenced by DNA hypermethylation in oral cancer. Cancer Res 68: 2094-2105, 2008.

15. Lewis A, Mitsuya K, Umlauf D, et al: Imprinting on distal chromosome 7 in the placenta involves repressive histone methylation independent of DNA methylation. Nat Genet 36: 1291-1295, 2004

16. Balaguer F, Link A, Lozano JJ, et al: Epigenetic silencing of miR-137 is an early event in colorectal carcinogenesis. Cancer Res 70: 6609-6618, 2011.

17. Bandres E, Agirre X, Bitarte N, et al: Epigenetic regulation of microRNA expression in colorectal cancer. Int J Cancer 125: $2737-2743,2009$.
18. Toyota M, Suzuki H, Sasaki Y, et al: Epigenetic silencing of microRNA-34b/c and B-cell translocation gene 4 is associated with $\mathrm{CpG}$ island methylation in colorectal cancer. Cancer Res 68: 4123-4132, 2008

19. Lujambio A, Ropero S, Ballestar E, et al: Genetic unmasking of an epigenetically silenced microRNA in human cancer cells. Cancer Res 67: 1424-1429, 2007.

20. Chiyomaru T, Enokida H, Kawakami K, et al: Functional role of LASP1 in cell viability and its regulation by microRNAs in bladder cancer. Urol Oncol: Sep 14, 2010 (Epub ahead of print).

21. Kojima S, Chiyomaru T, Kawakami K, et al: Tumour suppressors miR-1 and miR-133a target the oncogenic function of purine nucleoside phosphorylase (PNP) in prostate cancer. $\mathrm{Br} \mathrm{J}$ Cancer 106: 405-413, 2012.

22. Nohata N, Hanazawa T, Kikkawa N, et al: Identification of novel molecular targets regulated by tumor suppressive miR-1/ miR-133a in maxillary sinus squamous cell carcinoma. Int $\mathbf{J}$ Oncol 39: 1099-1107, 2011.

23. Rao PK, Missiaglia E, Shields L, et al: Distinct roles for miR-1 and miR-133a in the proliferation and differentiation of rhabdomyosarcoma cells. FASEB J 24: 3427-3437, 2010.

24. Yoshino H, Chiyomaru T, Enokida H, et al: The tumoursuppressive function of miR-1 and miR-133a targeting TAGLN2 in bladder cancer. Br J Cancer 104: 808-818, 2011.

25. Datta J, Kutay H, Nasser MW, et al: Methylation mediated silencing of MicroRNA-1 gene and its role in hepatocellular carcinogenesis. Cancer Res 68: 5049-5058, 2008.

26. Chen C, Ridzon DA, Broomer AJ, et al: Real-time quantification of microRNAs by stem-loop RT-PCR. Nucleic Acids Res 33: e179, 2005.

27. Zhang Y, Ye Y, Shen D, et al: Identification of transgelin-2 as a biomarker of colorectal cancer by laser capture microdissection and quantitative proteome analysis. Cancer Sci 101: 523-529, 2010.

28. Zhao L, Wang H, Liu C, et al: Promotion of colorectal cancer growth and metastasis by the LIM and SH3 domain protein 1. Gut 59: 1226-1235, 2010.

29. Kuwabara Y, Ono K, Horie T, et al: Increased microRNA-1 and microRNA-133a levels in serum of patients with cardiovascular disease indicate myocardial damage. Circ Cardiovasc Genet 4: 446-454, 2011.

30. He B, Xiao J, Ren AJ, et al: Role of miR-1 and miR-133a in myocardial ischemic postconditioning. J Biomed Sci 18: 22, 2011.

31. Bostjancic E, Zidar N, Stajer D and Glavac D: MicroRNAs miR-1, miR-133a, miR-133b and miR-208 are dysregulated in human myocardial infarction. Cardiology 115: 163-169, 2010.

32. Hudson RS, Yi M, Esposito D, et al: MicroRNA-1 is a candidate tumor suppressor and prognostic marker in human prostate cancer. Nucleic Acids Res 40: 3689-3703, 2012.

33. Migliore C, Martin V, Leoni VP, et al: MiR-1 downregulation cooperates with MACC1 in promoting MET overexpression in human colon cancer. Clin Cancer Res 18: 737-747, 2012.

34. Nasser MW, Datta J, Nuovo G, et al: Down-regulation of micro-RNA-1 (miR-1) in lung cancer. Suppression of tumorigenic property of lung cancer cells and their sensitization to doxorubicin-induced apoptosis by miR-1. J Biol Chem 283: 33394-33405, 2008.

35. Sarver AL, French AJ, Borralho PM, et al: Human colon cancer profiles show differential microRNA expression depending on mismatch repair status and are characteristic of undifferentiated proliferative states. BMC Cancer 9: 401, 2009.

36. Yan D, Dong Xda E, Chen X, et al: MicroRNA-1/206 targets c-Met and inhibits rhabdomyosarcoma development. J Biol Chem 284: 29596-29604, 2009.

37. Yoshino H, Enokida H, Chiyomaru T, et al: Tumor suppressive microRNA-1 mediated novel apoptosis pathways through direct inhibition of splicing factor serine/arginine-rich 9 (SRSF9/ SRp30c) in bladder cancer. Biochem Biophys Res Commun 417: 588-593, 2012.

38. Kano M, Seki N, Kikkawa N, et al: miR-145, miR-133a and miR-133b: Tumor-suppressive miRNAs target FSCN1 in esophageal squamous cell carcinoma. Int J Cancer 127: 2804-2814, 2010.

39. Ma Y, Zhang P, Yang J, Liu Z, Yang Z and Qin H: Candidate microRNA biomarkers in human colorectal cancer: Systematic review profiling studies and experimental validation. Int J Cancer 130: 2077-2087, 2012 
40. Moriya Y, Nohata N, Kinoshita T, et al: Tumor suppressive microRNA-133a regulates novel molecular networks in lung squamous cell carcinoma. J Hum Genet 57: 38-45, 2012.

41. Nohata N, Hanazawa T, Kikkawa N, et al: Caveolin-1 mediates tumor cell migration and invasion and its regulation by miR-133a in head and neck squamous cell carcinoma. Int J Oncol 38: 209-217, 2011.

42. Wu ZS, Wang CQ, Xiang R, et al: Loss of miR-133a expression associated with poor survival of breast cancer and restoration of miR-133a expression inhibited breast cancer cell growth and invasion. BMC Cancer 12: 51, 2012.
43. Rho JH, Roehrl MH and Wang JY: Tissue proteomics reveals differential and compartment-specific expression of the homologs transgelin and transgelin-2 in lung adenocarcinoma and its stroma. J Proteome Res 8: 5610-5618, 2009.

44. Leung WK, Ching AK, Chan AW, et al: A novel interplay between oncogenic PFTK1 protein kinase and tumor suppressor TAGLN2 in the control of liver cancer cell motility. Oncogene 30: 4464-4475, 2011.

45. Suzuki H, Takatsuka S, Akashi H, et al: Genome-wide profiling of chromatin signatures reveals epigenetic regulation of MicroRNA genes in colorectal cancer. Cancer Res 71: 5646$5658,2011$. 